\title{
PROPERTIES AND EVOLUTION OF THE REDBACK MILLISECOND PULSAR BINARY PSR J2129-0429
}

\author{
Eric C. Bellm ${ }^{1}$, David L. Kaplan ${ }^{2}$, Rene P. Breton ${ }^{3,4}$, E. Sterl Phinney ${ }^{1}$, Varun B. Bhalerao ${ }^{5}$, Fernando Camilo ${ }^{6}$, \\ Sumit Dahal $^{7}$, S. G. Djorgovski ${ }^{1}$, Andrew J. Drake ${ }^{1}$, J. W. T. Hessels ${ }^{8,9}$, Russ R. Laher ${ }^{10}$, David B. Levitan ${ }^{1}$, \\ Fraser Lewis $^{11,12}$, Ashish A. Mahabal ${ }^{1}$, Eran O. OfeK ${ }^{13}$, Thomas A. Prince ${ }^{1}$, Scott M. Ransom ${ }^{14}$, \\ Mallory S. E. Roberts ${ }^{15}$, David M. Russell ${ }^{7}$, Branimir Sesar ${ }^{16}$, Jason A. Surace ${ }^{10}$, and Sumin Tang ${ }^{1}$ \\ ${ }^{1}$ Cahill Center for Astronomy and Astrophysics, California Institute of Technology, Pasadena, CA 91125, USA; ebellm@ caltech.edu \\ ${ }^{2}$ Department of Physics, University of Wisconsin-Milwaukee, Milwaukee, WI 53201, USA \\ ${ }^{3}$ Jodrell Bank Centre for Astrophysics, Alan Turing Building, School of Physics and Astronomy, \\ The University of Manchester, Oxford Road, Manchester, M13 9PL, UK \\ ${ }^{4}$ School of Physics and Astronomy, University of Southampton, Southampton, SO17 1BJ, UK \\ ${ }^{5}$ Inter University Centre for Astronomy and Astrophysics, PO Bag 4, Ganeshkhind, Pune 411007, India \\ ${ }^{6}$ Columbia Astrophysics Laboratory, Columbia University, New York, NY 10027, USA \\ ${ }^{7}$ New York University Abu Dhabi, P.O. Box 129188, Abu Dhabi, UAE \\ ${ }^{8}$ ASTRON, the Netherlands Institute for Radio Astronomy, Postbus 2, 7990 AA, Dwingeloo, The Netherlands \\ ${ }^{9}$ Anton Pannekoek Institute for Astronomy, University of Amsterdam, Science Park 904, 1098 XH Amsterdam, The Netherlands \\ ${ }^{10}$ Infrared Processing and Analysis Center, California Institute of Technology, Pasadena, CA 91125, USA \\ ${ }^{11}$ Faulkes Telescope Project, School of Physics and Astronomy, Cardiff University, 5 The Parade, Cardiff, CF24 3AA, Wales, UK \\ 12 Astrophysics Research Institute, Liverpool John Moores University, IC2, Liverpool Science Park, 146 Brownlow Hill, Liverpool L3 5RF, UK \\ ${ }^{13}$ Benoziyo Center for Astrophysics, Faculty of Physics, Weizmann Institute of Science, Rehovot 76100, Israel \\ ${ }^{14}$ National Radio Astronomy Observatory, 520 Edgemont Road, Charlottesville, Virginia 22903-2475, USA \\ ${ }^{15}$ Eureka Scientific Inc., 2452 Delmer Street, Suite 100, Oakland, California 94602-3017, USA \\ ${ }^{16}$ Max Planck Institute for Astronomy, Königstuhl 17, D-69117 Heidelberg, Germany \\ Received 2015 October 4; accepted 2015 December 1; published 2016 January 14
}

\begin{abstract}
PSR J2129-0429 is a "redback" eclipsing millisecond pulsar binary with an unusually long $15.2 \mathrm{hr}$ orbit. It was discovered by the Green Bank Telescope in a targeted search of unidentified Fermi gamma-ray sources. The pulsar companion is optically bright (mean $m_{R}=16.6 \mathrm{mag}$ ), allowing us to construct the longest baseline photometric data set available for such a system. We present 10 years of archival and new photometry of the companion from the Lincoln Near-Earth Asteroid Research Survey, the Catalina Real-time Transient Survey, the Palomar Transient Factory, the Palomar 60 inch, and the Las Cumbres Observatory Global Telescope. Radial velocity spectroscopy using the Double-Beam Spectrograph on the Palomar 200 inch indicates that the pulsar is massive: $1.74 \pm 0.18 M_{\odot}$. The G-type pulsar companion has mass $0.44 \pm 0.04 M_{\odot}$, one of the heaviest known redback companions. It is currently $95 \pm 1 \%$ Roche-lobe filling and only mildly irradiated by the pulsar. We identify a clear $13.1 \mathrm{mmag}^{-1}$ secular decline in the mean magnitude of the companion as well as smaller-scale variations in the optical light curve shape. This behavior may indicate that the companion is cooling. Binary evolution calculations indicate that PSR J2129-0429 has an orbital period almost exactly at the bifurcation period between systems that converge into tighter orbits as black widows and redbacks and those that diverge into wider pulsar-white dwarf binaries. Its eventual fate may depend on whether it undergoes future episodes of mass transfer and increased irradiation.
\end{abstract}

Key words: pulsars: individual (PSR J2129-0429)

\section{INTRODUCTION}

Millisecond pulsars (MSPs) eclipsed by material from their low-mass binary companions may provide a major path to the production of isolated MSPs: after spinning up the pulsar through accretion (Alpar et al. 1982; Radhakrishnan \& Srinivasan 1982; Bhattacharya \& van den Heuvel 1991), the pulsar wind may ablate away the donor (Kluzniak et al. 1988; Phinney et al. 1988; van den Heuvel \& van Paradijs 1988). This cannibalism suggested the name "Black Widow" upon the discovery of the first example, which had a companion mass of only a few percent $M_{\odot}$ (Fruchter et al. 1988). Later observations and theoretical work suggested that the ablation rate for the first Black Widow system might be too slow to evaporate its companion in a Hubble time, however (Levinson \& Eichler 1991; Fruchter \& Goss 1992). Ablation may be more efficient in other systems (e.g., Bailes et al. 2011), such that the companion is completely destroyed or only a small remnant remains. Instabilities in very low mass companions (Deloye \& Bildsten 2003) or dynamical encounters in globular clusters
(King et al. 2003) provide alternative means to produce isolated MSPs.

Today, thanks to targeted radio and X-ray surveys of globular clusters and, more recently, in regions of the broader Galactic Field where Fermi-LAT has localized sources, more than 30 black widow systems are known (Roberts 2013, and references therein). Moreover, many "redback" systems ${ }^{17}$ with higher-mass, non-degenerate companions of $0.2-0.7 M_{\odot}$ have been discovered (Roberts 2013, and references therein; Crawford et al. 2013). The discovery of several redback systems that transition between accretion-powered low-mass $\mathrm{X}$-ray binary states and rotation-powered radio pulsar states has provided further support for the recycling scenario (Archibald et al. 2009; Papitto et al. 2013; Bassa et al. 2014; Patruno et al. 2014; Stappers et al. 2014). Irradiation feedback and accretion disk instabilities may be responsible for the relatively short timescale transitions (years) observed between accreting and detached states (Benvenuto et al. 2015). Current models

\footnotetext{
$\overline{{ }^{17} \text { Redback spiders are the Australian cousins of Black Widow spiders. }}$
} 
Table 1

Summary of Photometric Observations

\begin{tabular}{|c|c|c|c|c|c|c|}
\hline Telescope & Filter & Start Date & End Date & Exposure (s) & Number & Precision (mmag) \\
\hline LINEAR & open & 2003 May 30 & 2011 Oct 29 & $3-18$ & 470 & 30 \\
\hline CRTS & open & 2005 May 12 & 2014 May 5 & 30 & 365 & 76 \\
\hline P48 & Mould $R$ & 2009 Jun 26 & 2014 Oct 11 & 60 & 116 & 14 \\
\hline P60 & $g^{\prime}$ & 2011 Nov 10 & 2012 Jun 14 & 60,120 & 41 & 26 \\
\hline P60 & $r^{\prime}$ & 2011 Nov 10 & 2012 Jun 14 & 60,120 & 43 & 20 \\
\hline P60 & $i^{\prime}$ & 2011 Nov 10 & 2012 Jun 14 & 60,120 & 50 & 18 \\
\hline LCOGT $1 \& 2 \mathrm{~m}$ & $g^{\prime}$ & 2014 Oct 26 & 2014 Nov 22 & $100-200$ & 25 & 29 \\
\hline LCOGT $1 \& 2 \mathrm{~m}$ & $r^{\prime}$ & 2014 Oct 26 & 2014 Nov 22 & $100-200$ & 25 & 20 \\
\hline LCOGT $1 \& 2 \mathrm{~m}$ & $i^{\prime}$ & 2014 Oct 26 & 2014 Nov 22 & $100-200$ & 23 & 28 \\
\hline LCOGT $1 \& 2 \mathrm{~m}$ & Pan-STARRS $Z$ & 2014 Oct 26 & 2014 Nov 22 & $100-200$ & 13 & 69 \\
\hline
\end{tabular}

disagree as to whether redbacks and black widows are distinct populations that evolve separately due to bimodal evaporation efficiency (Chen et al. 2013) or whether black widow systems are an endpoint in the evolution of some redback systems (Benvenuto et al. 2014).

In the optical bands, black widow and redback binaries show large-amplitude variability due to effects such as the ellipsoidal modulation of the near-Roche filling companion, dayside and nightside temperature differentials, and gravity darkening. The apparent magnitudes of these systems vary considerably with distance and the intrinsic luminosity of their companions. With their larger companions and relative proximity, redbacks in the Galactic Field are the brightest of these systems in the optical band, allowing for detailed photometric and spectroscopic monitoring with moderate aperture telescopes (e.g., Breton et al. 2013; Li et al. 2014; Schroeder \& Halpern 2014). MSP binaries in the Galactic Field are also useful tracers of binary evolution, as unlike systems in globular clusters they are unlikely to experience dynamical exchange encounters.

With a radio timing solution and an optical radial velocity amplitude from the companion, it is possible to measure the mass ratio of the pulsar and its companion. While expected theoretically (e.g., Phinney \& Kulkarni 1994; Benvenuto et al. 2014), the neutron star masses in the black widow and redback systems measured to date have largely proven to be heavier than $1.4 M_{\odot}$ (van Kerkwijk et al. 2011; Deller et al. 2012; Romani et al. 2012; Crawford et al. 2013). Measurement of pulsar masses in these systems thus provides a valuable expansion of the sample needed to understand the recycling process. While the most precise measurements of the masses of high-mass neutron stars have been obtained through measurement of Shapiro delay (Nice et al. 2005; Demorest et al. 2010; Freire et al. 2011), discovery of additional heavy neutron stars in pulsar binaries of various types (e.g., Bassa et al. 2006; Antoniadis et al. 2013; Kaplan et al. 2013; Strader et al. 2015) can also inform studies of the equation of state of neutron stars (for a review, see Lattimer 2012).

PSR J2129-0429 is a redback system with a bright (mean $m_{R}=16.6$ ) optical counterpart. The gamma-ray counterpart to PSR J2129-0429 appeared in the first Fermi source catalog as 1FGL J2129.8-0427 (Abdo et al. 2010) and in subsequent versions as 2FGL J2129.8-0428 (Nolan et al. 2012) and 3FGL J2129.6-0427 (The Fermi-LAT Collaboration 2015). The Fermi spectrum does not show significant curvature, making it difficult to firmly classify as a pulsar based on the gamma-ray data alone (e.g., Ackermann et al. 2012).

Nevertheless, a radio survey of the 1FGL error box with the Green Bank Telescope identified a pulsar counterpart with a spin period of $7.62 \mathrm{~ms}$ (Hessels et al. 2011). The dispersion measure of $16.9 \mathrm{pc} \mathrm{cm}^{-3}$ implies a distance of $0.9 \mathrm{kpc}$ using the NE2001 model (Cordes \& Lazio 2002). Phase-folded X-ray observations of the system show an unusual double-peaked phase profile from the intra-binary shock, suggesting a compact but structured emission region (Roberts et al. 2015).

In this paper we present optical photometry and spectroscopy of the PSR J2129-0429 binary system. In Section 2 we describe the observations. We perform fits to the radial velocity amplitude in Section 3 and to the system geometry in Section 4. In Section 5 we present evidence for secular evolution in the light curve. We close with a discussion of the evolution of PSR J2129-0429 (Section 6).

Throughout the paper, we use the convention that the zero point of orbital phase occurs when the companion is between the pulsar and the Earth (companion inferior conjunction). The Time of Ascending Node is accordingly at phase 0.75 in this convention.

\section{OBSERVATIONS}

\subsection{Photometry}

Using the localization of PSR J2129-0429 provided by a preliminary radio-derived pulsar timing ephemeris $\left(21^{\mathrm{h}} 29^{\mathrm{m}} 45^{\mathrm{s}} .039 \pm 0.001, \quad-04^{\circ} 29^{\prime} 05.59 \pm 00^{\prime \prime} 08 \quad(\mathrm{~J} 2000)\right.$; P. Bangale et al. 2015, in preparation), we found a bright $\left(m_{R} \sim 16.5\right)$ optical counterpart in the databases of several time-domain surveys that varied at the orbital period of $15.245 \mathrm{hr}$. We also acquired new multicolor phase-resolved observations to enable detailed light curve modeling.

The optical counterpart of PSR J2129-0429 was repeatedly detected in imaging conducted by the Palomar Transient Factory (PTF) (Law et al. 2009; Rau et al. 2009) on the Palomar Samuel Oschin 48 inch Schmidt $(\mathrm{P} 48)$ at $21^{\mathrm{h}} 29^{\mathrm{m}} 45^{\mathrm{s}} .06,-04^{\circ} 29^{\prime} 06^{\prime \prime} 8$ (J2000). ${ }^{18}$ We obtained calibrated aperture photometry for PSR J2129-0429 from the standard PTF photometric pipeline, which includes image reduction (Laher et al. 2014), photometric calibration (Ofek et al. 2012) to the Sloan Digital Sky Survey (York et al. 2000), and a relative photometry correction for each image to remove any residual scalar offsets in the zero-points due to non-photometric conditions (c.f. Ofek et al. 2011, Appendix A). We added a systematic error of $0.01 \mathrm{mag}$ in quadrature with the pipeline-generated photometric errors so that

\footnotetext{
$\overline{18}$ Typical PTF absolute astrometric errors are $0 . \prime 1-0 . ! 2$ rms. The 1 !" 25 distance of the radio position may be due to covariances between the orbital derivatives and spindown in the relatively short radio timing baseline. The SDSS DR12 (Alam et al. 2015) position of the object $\left(21^{\mathrm{h}} 29^{\mathrm{m}} 45^{\mathrm{s}} .05\right.$, $-04^{\circ} 29^{\prime} 06^{\prime \prime} 83$ ) is consistent with the PTF position.
} 
Table 2

Summary of Spectroscopic Observations

\begin{tabular}{|c|c|c|c|c|}
\hline Date (UTC) & $\begin{array}{c}\text { Epoch at Barycenter } \\
\text { (TDB MJD) }\end{array}$ & Orbital Phase & $\begin{array}{c}\text { Aperture } \\
(\operatorname{arcsec})\end{array}$ & $\begin{array}{c}\text { Exposure Time } \\
\text { (s) }\end{array}$ \\
\hline 2011 Dec 27 & 55922.07355 & 0.02 & 1.0 & 900 \\
\hline 2012 May 29 & 56076.45451 & 0.06 & 1.0 & 900 \\
\hline 2012 Sep 20 & 56190.20842 & 0.13 & 0.5 & 900 \\
\hline 2012 Sep 20 & 56190.25525 & 0.21 & 0.5 & 900 \\
\hline 2012 Sep 20 & 56190.30287 & 0.28 & 0.5 & 900 \\
\hline 2012 Sep 21 & 56191.30869 & 0.86 & 0.5 & 900 \\
\hline 2013 Sep 02 & 56445.45257 & 0.95 & 0.5 & 900 \\
\hline $2013 \mathrm{Jul} 04$ & 56477.47904 & 0.36 & 1.5 & 900 \\
\hline $2013 \mathrm{Jul} 04$ & 56477.48975 & 0.38 & 1.5 & 900 \\
\hline 2013 Jul 04 & 56477.50047 & 0.40 & 1.5 & 900 \\
\hline
\end{tabular}

Note. All observations were conducted with the Double Beam Spectrograph of the Palomar 200 inch Hale Telescope. Gratings used were 600 lines $/ \mathrm{mm}$ blazed at $4000 \AA$ (blue side) and 316 lines/mm blazed at $7500 \AA$ (red side) for all dates except 2013 July 4, when the 1200/5000 and 1200/7100 gratings were used.

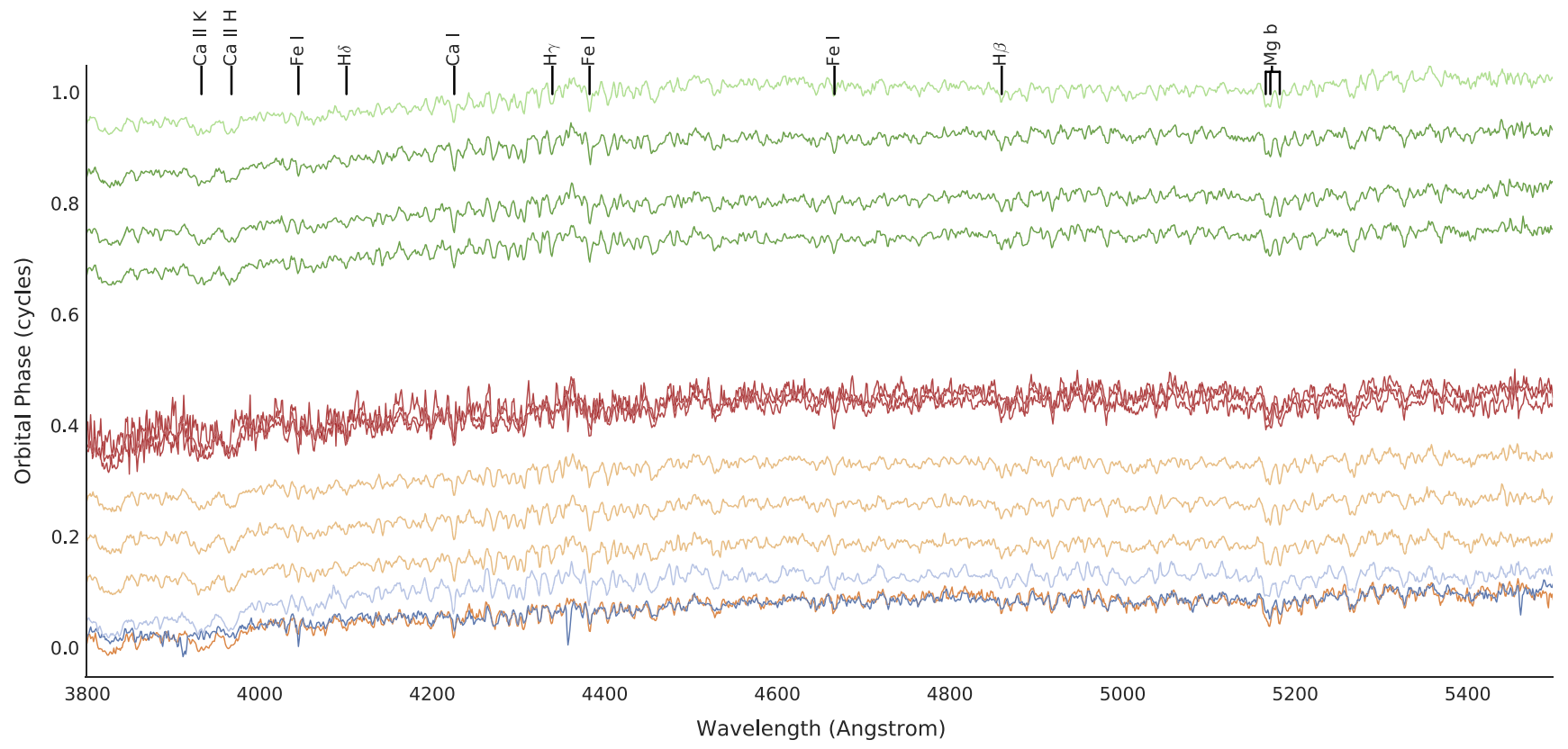

Figure 1. Blue-arm DBSP spectra of the PSR J2129-0429 companion ordered by pulsar orbital phase. Spectra are adjusted to zero velocity. Spectra obtained within one night of observing are plotted in the same color; Table 2 lists the orbital phase of each spectrum.

a constant fit to a nearby (53." 6 separation) comparison star $\left(21^{\mathrm{h}} 29^{\mathrm{m}} 46^{\mathrm{s}} .9,-04^{\circ} 28^{\prime} 20^{\prime \prime} .5, m_{R}=16.6 \mathrm{mag}\right)$ had reduced chisquared $\chi_{\nu}^{2}=1$.

PSR J2129-0429 is also present in data from the Catalina Real-time Transient Survey (CRTS; Drake et al. 2009) catalog and in its associated catalog of periodic sources (Drake et al. 2014). The Catalina images are spaced ten minutes apart to look for asteroid motion. The magnitudes are obtained by running SExtractor (Bertin \& Arnouts 1996) on the images and

\footnotetext{
19 More details for the CRTS Second Data Release (DR2) magnitudes can be found at http://nesssi.cacr.caltech.edu/DataRelease/FAQ2.html\#calib. The set of observations used here included dates outside the DR2 release. Magnitudes from these observations are taken from the transient pipeline and lack the small frame offsets applied in DR2.
}

then calibrating them to the $V$ band $^{19}$, as the raw CCD images are unfiltered.

Additionally, the system was imaged by the Lincoln NearEarth Asteroid Research survey (LINEAR; Stokes et al. 2000; Sesar et al. 2011). We performed point-spread function photometry on the images and photometrically calibrated the images to SDSS.

We acquired targeted phase-resolved observations of PSR J2129-0429 in $g^{\prime}, r^{\prime}$, and $i^{\prime}$ using the CCD camera on the roboticized Palomar 60 inch telescope (P60; Cenko et al. 2006) between 2011 November and 2012 June. Basic image reductions were performed by the automated pipeline. We obtained aperture photometry for the source and companion stars using SExtractor and calibrated the photometry to SDSS. 


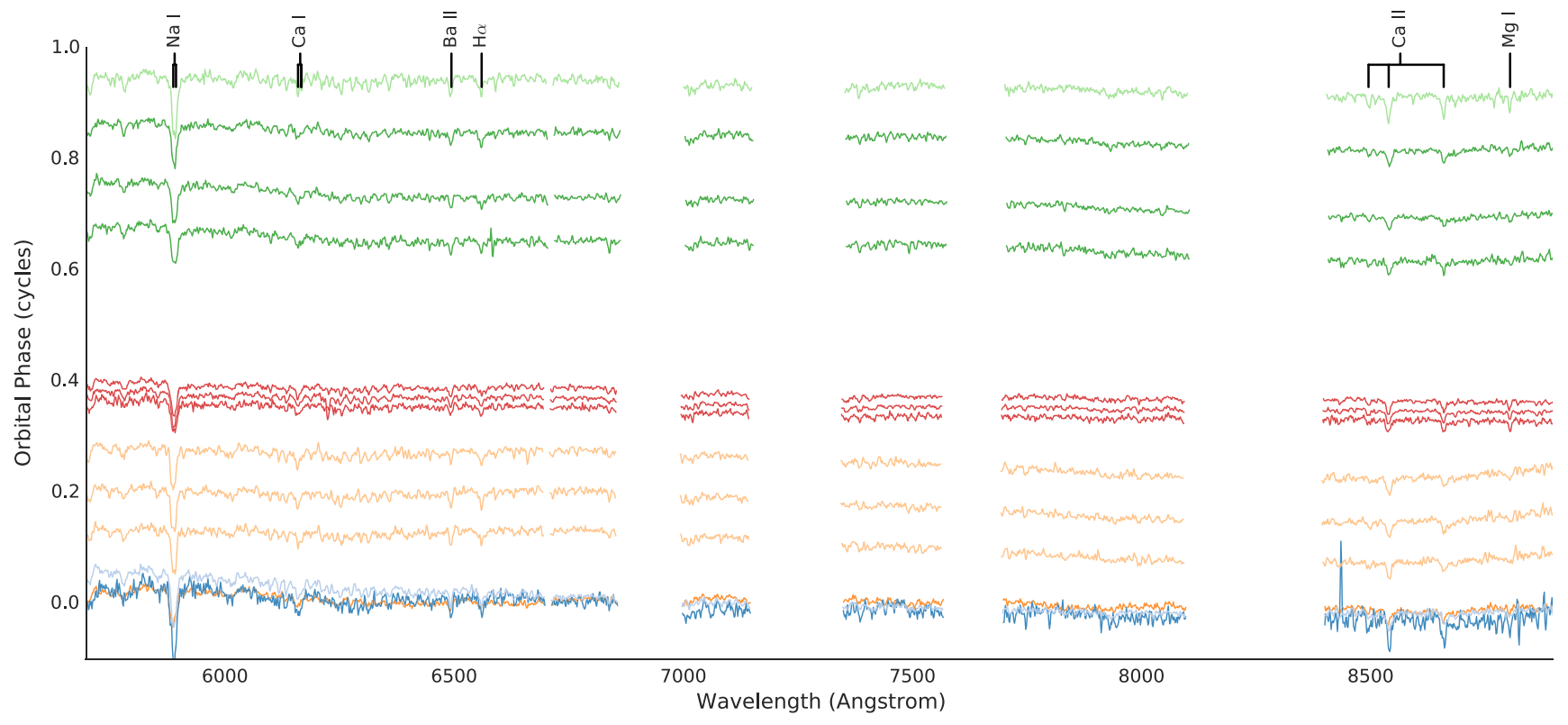

Figure 2. Red-arm DBSP spectra of the PSR J2129-0429 companion ordered by pulsar orbital phase. Colors are as in Figure 1. Regions of telluric absorption are masked, as is a small region near $6707 \AA$ affected by a dome lamp fluorescence feature.

We obtained additional $g^{\prime}, r^{\prime}, i^{\prime}$ and $z^{\prime}$ imaging of PSR J2129 -0429 using the Las Cumbres Observatory Global Telescope (LCOGT) Network over a period of a month starting 2014 October 26. Observations were made using the $1 \mathrm{~m}$ LCOGT network sites at Siding Spring Observatory, Australia, Cerro Tololo Inter-American Observatory, Chile, McDonald Observatory, USA and SAAO, South Africa, as well as the $2 \mathrm{~m}$ Faulkes Telescopes at Haleakala Observatory, Maui, Hawaii, USA and Siding Spring Observatory, Australia. The data reduction was performed using LCOGT's automatic pipeline and aperture photometry was conducted with IRAF version 2.16.1 (Tody 1986) and calibrated to SDSS using four bright, unsaturated stars visible in all images.

All times were corrected to the solar system barycenter. The photometric observations are summarized in Table 1.

\subsection{Spectroscopy}

We obtained spectra of PSR J2129-0429 using the Palomar 200 inch Hale telescope and the Double-Beam Spectrograph (DBSP; Oke \& Gunn 1982) using the new red camera (Rahmer et al. 2012). Table 2 lists the observation parameters. We reduced data from both arms of the spectrograph using a custom PyRAF-based pipeline. ${ }^{20}$ The pipeline performs standard image processing and spectral reduction procedures, including bias subtraction, flat-field correction, wavelength calibration, optimal spectral extraction, and flux calibration. To account for instrument flexure in individual science spectra, we subtract an average wavelength offset computed from known sky lines on a per-frame basis (Sesar et al. 2013). The rms scatter of the corrected sky line positions provides an estimate of the uncertainty in the wavelength solution, which we add in quadrature with the template fitting uncertainty when computing radial velocities (Section 3). The mean uncertainty for these observations was $6.2 \mathrm{~km} \mathrm{~s}^{-1}$ at $4750 \AA$ on the blue side and $8.6 \mathrm{~km} \mathrm{~s}^{-1}$ at $7400 \AA$ on the red side. Figures 1 and 2 show the reduced spectra ordered by pulsar orbital phase.

\footnotetext{
${ }^{20}$ https://github.com/ebellm/pyraf-dbsp
}

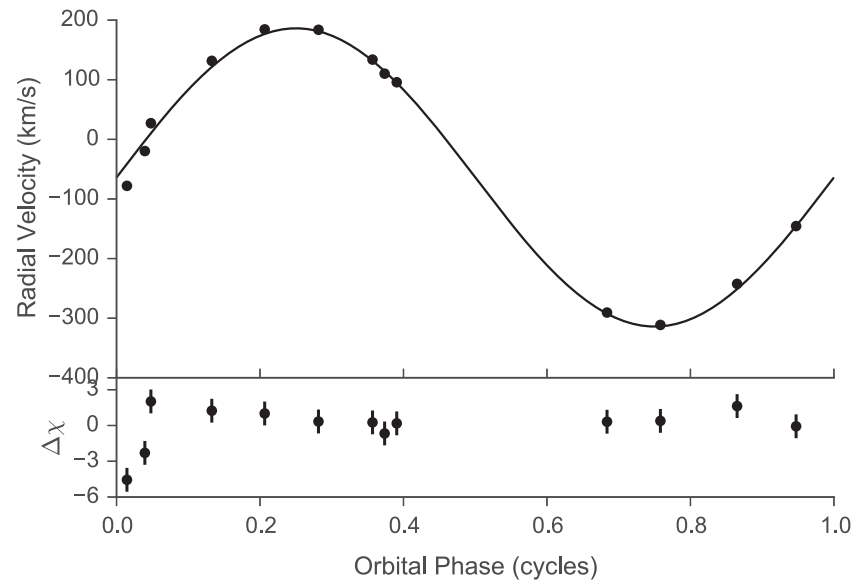

Figure 3. Radial velocity values, best-fit curve, and sigma residuals for PSR J2129-0429 from the DBSP red arm data. Error bars are comparable to the symbol size. The best fit amplitude is $K=250.0 \pm 3.7 \mathrm{~km} \mathrm{~s}^{-1}$ and the systemic velocity is $\gamma=-63.9 \pm 2.4 \mathrm{~km} \mathrm{~s}^{-1}$.

The spectra indicate that the companion of PSR J2129 -0429 is non-degenerate, with features resembling a G-type star. Some variation in the line strengths is observed. From the few observations we have that are repeated near phase 0 , there appears to be stochastic variability in addition to phasedependent differences (Figures 1 and 2).

\section{RADIAL VELOCITY}

We obtained radial velocity measurements from our spectra using a template-fitting approach as implemented in Bhalerao et al. (2012). We fit flux-calibrated spectra with the stellar atmosphere models of Munari et al. (2005), using a linear polynomial to account for any residual fluxing errors. We included an extinction coefficient of $A_{V}=0.10 \mathrm{mag}$ (Schlafly \& Finkbeiner 2011). We fit the red-arm data from $5700-8900 \AA$, omitting telluric bands at $6860-7000 \AA$, 7570-7700, $7150-7350 \AA$, and $8100-8400 \AA$. The best-fit 


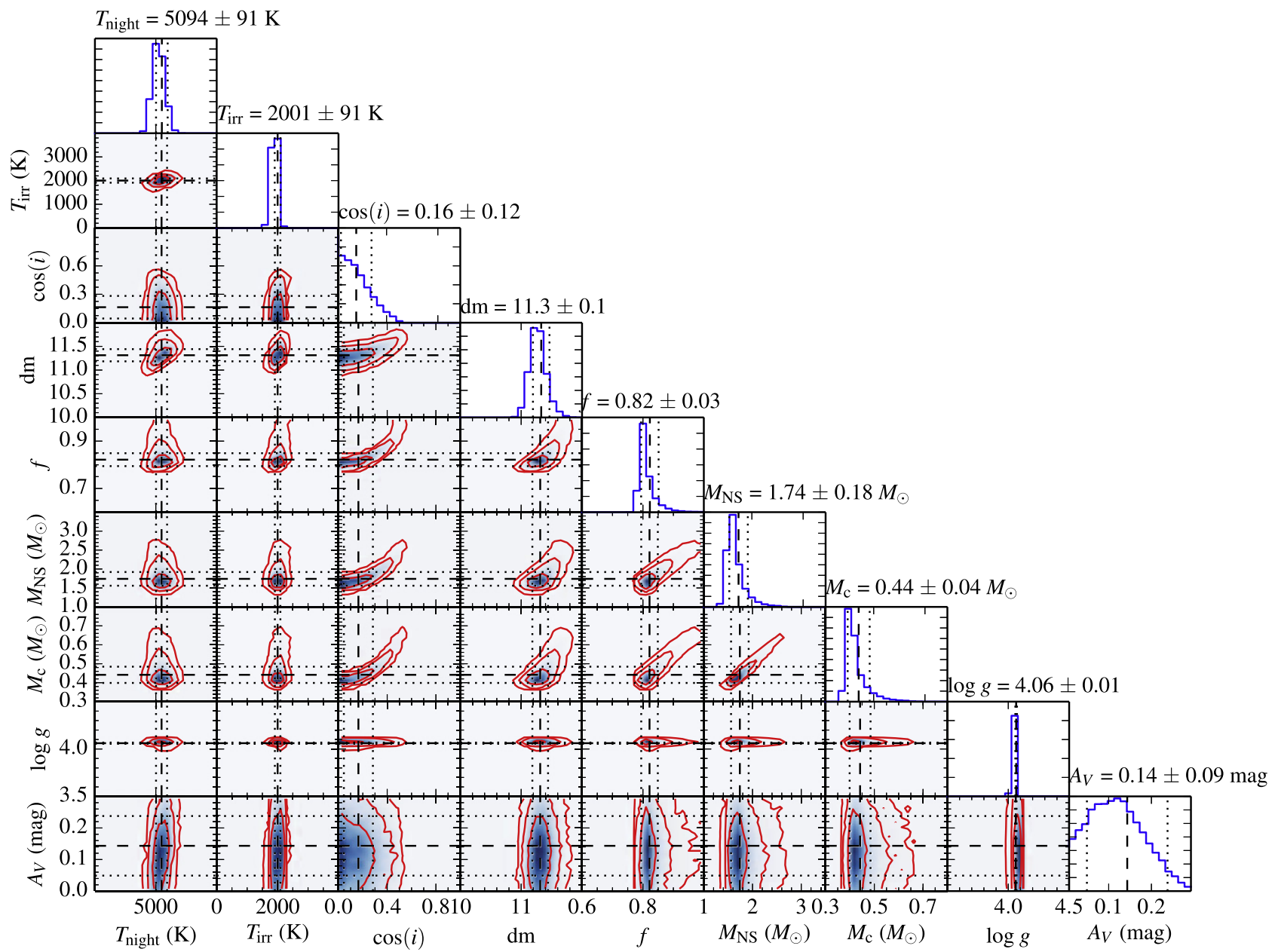

Figure 4. Joint two-dimensional posterior probability functions from the MCMC analysis. We show 1-, 2-, and 3- $\sigma$ joint probability contours for each pair of parameters as well as the marginalized one-dimensional posterior pdfs for each parameter. See Table 3 for details.

atmosphere model at all phases had $T=5750 \mathrm{~K}, \log g=5.0$, solar metallicity, and $100 \mathrm{~km} \mathrm{~s}^{-1}$ rotational broadening. (These values are broadly consistent with the values we derive from binary modeling in Section 4 and our assumption of corotation.) By stepping the model through a grid of wavelength offsets, we determined the radial velocity at each epoch. A quadratic fit to the $\chi^{2}$ surface provided $1 \sigma$ error estimates, which we added in quadrature with the wavelength calibration uncertainties obtained in Section 2.2. Finally, we converted the observed radial velocities to the solar system barycenter using Python routines derived from the Standards of Fundamental Astronomy Library (IAU SOFA Board 2015). The $\chi^{2} /$ dof for the fit is $11.6 / 11$. Figure 3 shows the resulting radial velocities and best-fit radial velocity amplitude using the radio ephemeris $\left(P_{\text {orb }}=0.63522741310 \pm 3.3 \times 10^{-10}\right.$ days; $\left.\mathrm{TASC}=55702.111161463 \pm 9.8 \times 10^{-8}\right) .{ }^{21} \mathrm{We}$ find a radial velocity amplitude $K=250.0 \pm 3.7 \mathrm{~km} \mathrm{~s}^{-1}$. Together with the measured projected semimajor axis (1.85 lt-s) from pulsar timing we find a mass ratio $q=3.93 \pm 0.06$. Because the irradiation of the companion by the pulsar is modest and the mass ratio less extreme than in black widow systems (e.g.,

\footnotetext{
${ }^{21}$ We do not include radio-derived orbital period derivatives because the time baseline for our optical data extends well beyond the radio timing interval, which would lead to significant extrapolation errors from the higher-order period derivatives.
}

van Kerkwijk et al. 2011), the correction of the measured center of light RV amplitude to the center of mass is small $(\sim 0.2 \%)$, removing a potential source of systematic error. We nevertheless model this correction self-consistently in our binary fitting in the next section.

\section{BINARY FITTING}

We fit the available multicolor photometry from P60, and LCOGT as well as the 2009-2010 PTF data to a model of a distorted, irradiated binary companion using Icarus (Breton et al. 2013). This fitting computed filter-dependent photometry for a companion including the effects of irradiation and ellipsoidal modulation, which enable us to constrain the inclination, the masses of the binary members, the distance, the degree of irradiation, etc. Our fitting made use of prior probability distributions on the distance, extinction, and radial velocity amplitude. The distance prior was based on the dispersion measure (DM) distance of $900 \mathrm{pc}$ (Cordes \& Lazio 2002) with a conservative estimated uncertainty of $50 \%( \pm 450 \mathrm{pc})$. The radial velocity amplitude prior was based on the measured radial velocity amplitude as well as the pulsar's projected semimajor axis, and we used $K=250 \pm 4 \mathrm{~km} \mathrm{~s}^{-1}$ as determined above. Finally we determined a prior on the extinction $A_{V}$ based on the measured X-ray 
Table 3

Best-fit Binary Parameter Values for PSR J2129-0429 Based on the MCMC Icarus Analysis

\begin{tabular}{|c|c|}
\hline Parameter & Value \\
\hline \multicolumn{2}{|c|}{ Fitted Parameters } \\
\hline $\cos i^{\mathrm{a}}$ & $0.16 \pm 0.12$ \\
\hline$T_{\text {night }}^{\mathrm{b}}(\mathrm{K})$ & $5094 \pm 90$ \\
\hline$T_{\text {day }}{ }^{\mathrm{c}}(\mathrm{K})$ & $5124 \pm 92$ \\
\hline$K^{\mathrm{d}}\left(\mathrm{km} \mathrm{s}^{-1}\right)$ & $250.3 \pm 4.3$ \\
\hline $\mathrm{dm}^{\mathrm{e}}$ & $11.3 \pm 0.1$ \\
\hline$A_{V}^{\mathrm{f}}(\mathrm{mag})$ & $0.14 \pm 0.09$ \\
\hline$f^{\mathrm{e}}$ & $0.82 \pm 0.03$ \\
\hline$\chi^{2} / \mathrm{dof}$ & $558.9 / 328$ \\
\hline \multicolumn{2}{|c|}{ Derived Parameters } \\
\hline$d^{\mathrm{h}}(\mathrm{pc})$ & $1833 \pm 110$ \\
\hline $\log (g)^{\mathrm{i}}$ & $4.06 \pm 0.01$ \\
\hline$q^{\mathrm{j}}$ & $3.94 \pm 0.07$ \\
\hline$M_{\mathrm{c}}{ }^{\mathrm{k}}\left(M_{\odot}\right)$ & $0.44 \pm 0.04$ \\
\hline$M_{\mathrm{NS}}^{1}\left(M_{\odot}\right)$ & $1.74 \pm 0.18$ \\
\hline$i^{\mathrm{m}}(\mathrm{deg})$ & $80.5 \pm 7.0$ \\
\hline$T_{\text {irr }}{ }^{\mathrm{n}}(\mathrm{K})$ & $2001 \pm 91$ \\
\hline$\eta^{\mathrm{o}}(\%)$ & $3.0 \pm 0.6$ \\
\hline$R / R_{L}{ }^{\mathrm{p}}$ & $0.95 \pm 0.01$ \\
\hline
\end{tabular}

Notes.

${ }^{\text {a }}$ Cosine of the inclination angle $i$.

${ }^{\mathrm{b}}$ Night-side temperature of the companion (i.e., the side facing away from the pulsar.

${ }^{c}$ Day-side temperature of the companion (i.e., the side facing toward the pulsar.

${ }^{\mathrm{d}}$ Radial velocity amplitude of the companion.

e Distance modulus.

${ }^{\mathrm{f}} V$-band extinction.

${ }^{g}$ Ratio of the radius of the companion pointing to the pulsar to that of the $\mathrm{L} 1$ point.

histance.

${ }^{\mathrm{i}}$ Volume-averaged surface gravity of the companion.

${ }^{\mathrm{j}}$ Mass ratio.

${ }^{\mathrm{k}}$ Companion mass.

${ }^{1}$ Pulsar mass.

${ }^{\mathrm{m}}$ Inclination angle.

${ }^{\mathrm{n}}$ Irradiation temperature, $\left(T_{\text {day }}^{4}-T_{\text {night }}^{4}\right)^{1 / 4}$.

${ }^{\circ}$ Irradiation efficiency, $4 \pi a^{2} \sigma T_{\text {irr }}^{4} / \dot{E}$, where $a$ is the semimajor axis.

${ }^{\mathrm{p}}$ Ratio of the volume-averaged radius to the volume-averaged Roche lobe radius.

column density $N_{\mathrm{H}}$ and the observed $A_{V}\left(N_{\mathrm{H}}\right)$ relation:

$$
p\left(A_{V}\right)=0.65 \mathcal{N}(0.131,0.082)+0.35 \mathcal{N}(0.068,0.188)
$$

(M. S. E. Roberts et al. 2015, in preparation). Here, $\mathcal{N}(\mu, \sigma)$ represents a Gaussian probability distribution function (pdf) with mean $\mu$ and standard deviation $\sigma$; both pdfs were properly truncated so that $A_{V} \geqslant 0$. Otherwise, we assumed flat priors on $\cos i$ (where $i$ is the binary inclination), the nightside temperature $T_{\text {night }}$, the dayside temperature $T_{\text {day }}$, and the Roche-lobe filling factor $f$ (here $f=R_{\text {nose }} / R_{\mathrm{L} 1}$ is the ratio of the radius of the companion pointing to the pulsar to that of the $\mathrm{L} 1$ point).

We performed a Markov-Chain Monte Carlo (MCMC) analysis of the photometry, fitting for $\cos i, T_{\text {night }}, T_{\text {day }}, K, \mathrm{DM}$, $A_{V}$, and $f$, where $\mathrm{DM}=-2.5 \log _{10}(d / 10 \mathrm{pc})$ is the distance modulus. We included a $0.01 \mathrm{mag}$ systematic uncertainty on each measurement, and we also allowed for individual offsets

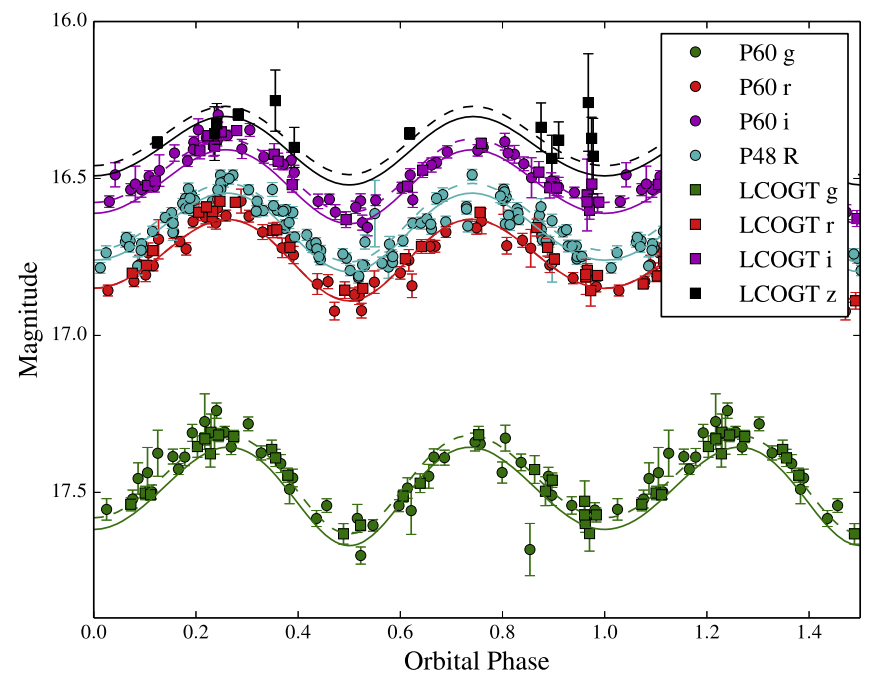

Figure 5. Best-fit light curve for PSR J2129-0429, using the P60, P48, and LCOGT photometry. The light curve is repeated 1.5 times for clarity. For each band we show the nominal model light curve (solid line) as well as the light curve with shifts to account for zero-point uncertainties (dashed line), all of which were $<0.04 \mathrm{mag}$.

for each telescope/filter combination with an assumed zero-point uncertainty of 0.05 mag. ${ }^{22}$ Through these we could also determine the individual masses $M_{\mathrm{NS}}$ and $M_{c}$, the companion's surface gravity $\log g$, as well as other related parameters such as the irradiation temperature $T_{\text {irr }}$ (defined as $\left.\left(T_{\text {day }}^{4}-T_{\text {night }}{ }^{4}\right)^{1 / 4}\right)$, the irradiation efficiency $\eta\left(4 \pi a^{2} \sigma T_{\text {irr }}{ }^{4} / \dot{E}\right)$ (where $\dot{E}$ is the pulsar spin-down luminosity $\dot{E}=4 \pi^{2} I \dot{P} P^{-3}$ ), and the volume-averaged Roche-lobe filling factor $R / R_{L}$. We assumed corotation, although for a binary period of $15 \mathrm{hr}$ the companion may not be tidally locked. We also assumed a gravity darkening coefficient $\beta=0.08$ (with effective temperature $\propto g^{\beta}$ corresponding to a fully convective envelope; Lucy 1967). We used 192 individual walkers to explore the parameter space and followed each for 20,000 iterations. We then rejected the first 200 iterations as "burn-in," and only included every 173rd point (based on observed autocorrelation lengths of $80-180$ iterations for individual parameters). The results of the MCMC are shown in Figure 4 and given in Table 3, where we list the mean of the posterior pdfs along with $68 \%$ confidence limits on individual parameters. Most of the two-dimensional marginalized pdfs are relatively well determined. The distance is a factor of 2 higher than the DM distance, although this is not uncommon for pulsars out of the Galactic Plane (Roberts 2011; Kaplan et al. 2013). We do not have a strong constraint on the inclination other than $\cos i<0.38$ at $95 \%$ confidence $\left(i>68^{\circ}\right)$. Even though the uncertainties on $T_{\text {night }}$ and $T_{\text {day }}$ overlap, there is actually a welldetermined irradiation efficiency as $T_{\text {night }}$ and $T_{\text {day }}$ are highly correlated. The best-fit light curve is given in Figure 5.

To explore the effects of our assumptions for corotation and gravity darkening, we did a limited series of experiments. We tried a corotation factor of 0.5 (rotation at half of the orbital speed) and a gravity darkening factor $\beta=0.04$. In both cases the majority of the fitted parameters remain within $1-\sigma$ of their

\footnotetext{
22 These normalization offsets remove the largest observed source of secular variation (Section 5). The fits are dominated by the P60 data, taken in 2011-2012. Little color or shape evolution is apparent in the more sparsely sampled 2014 LCOGT data.
} 
Table 4

Best-fit Coefficients (Equation (2)) for the Photometric Evolution of PSR J2129-0429 by Year

\begin{tabular}{|c|c|c|c|c|c|c|c|c|c|}
\hline Years & $m_{0}$ & $a_{1}$ & $a_{2}$ & $a_{3}$ & $a_{4}$ & $a_{5}$ & $\chi^{2}$ & dof & $\chi_{\nu}^{2}$ \\
\hline 2003-2004 & $16.545 \pm 0.003$ & $0.004 \pm 0.005$ & $0.114 \pm 0.004$ & $-0.010 \pm 0.005$ & $0.003 \pm 0.005$ & $0.018 \pm 0.005$ & 144.0 & 113 & 1.27 \\
\hline 2005-2006 & $16.573 \pm 0.003$ & $0.010 \pm 0.004$ & $0.113 \pm 0.005$ & $-0.013 \pm 0.004$ & $-0.006 \pm 0.005$ & $0.012 \pm 0.004$ & 177.5 & 205 & 0.87 \\
\hline 2007-2008 & $16.598 \pm 0.003$ & $-0.001 \pm 0.004$ & $0.117 \pm 0.004$ & $0.001 \pm 0.005$ & $0.013 \pm 0.005$ & $0.021 \pm 0.005$ & 211.7 & 184 & 1.15 \\
\hline 2009-2010 & $16.637 \pm 0.002$ & $-0.010 \pm 0.002$ & $0.117 \pm 0.002$ & $-0.014 \pm 0.002$ & $-0.004 \pm 0.002$ & $0.014 \pm 0.002$ & 228.0 & 220 & 1.04 \\
\hline 2011-2012 & $16.644 \pm 0.003$ & $-0.004 \pm 0.004$ & $0.117 \pm 0.003$ & $-0.017 \pm 0.003$ & $-0.022 \pm 0.003$ & $0.015 \pm 0.004$ & 127.4 & 142 & 0.90 \\
\hline 2013-2014 & $16.680 \pm 0.002$ & $-0.003 \pm 0.002$ & $0.106 \pm 0.002$ & $-0.005 \pm 0.003$ & $-0.022 \pm 0.002$ & $0.015 \pm 0.002$ & 116.0 & 117 & 0.99 \\
\hline
\end{tabular}

nominal values. Only the filling factor changes slightly (still by $<2 \sigma$ ), and this is largely to keep the shape of the photometric variations similar.

\section{LONG-TERM PHOTOMETRY}

In Figure 8 we show the LINEAR, CRTS, PTF, P60 $r^{\prime}$, and LCOGT $r^{\prime}$ photometry from 2003-2014. A secular evolution toward lower companion brightness is apparent in all surveys. We examined photometry from nearby stars of comparable brightness and found no long-term or phase-dependent trends.

To quantify this, we fit the data with an analytic model:

$$
\begin{aligned}
m(\phi)=m_{0}+a_{1} \cos 2 \pi \phi & +a_{2} \cos 4 \pi \phi+a_{3} \cos 6 \pi \phi \\
& +a_{4} \sin 2 \pi \phi+a_{5} \sin 4 \pi \phi
\end{aligned}
$$

where $m(\phi)$ is the magnitude at orbital phase $\phi$ and $\phi$ is in the range $0 \rightarrow 1$. The coefficient $a_{1}$ is dominated by irradiation, $a_{2}$ is dominated by ellipsoidal modulation, and $a_{3}$ accounts for residual distortion. Of the sine coefficients, $a_{4}$ captures relativistic beaming and $a_{5}$ fits residual distortions.

We fit for each coefficient $m_{0}, a_{1}, a_{2}, a_{3}, a_{4}, a_{5}$ in each time bin in Figure 8 after normalizing the values of $m_{0}$ to the PTF $R$ band. Parameter agreement between the different instruments is acceptable, as indicated by the reasonable goodness of fit $\left(\chi^{2} /\right.$ dof $\left.=1004.5 / 981\right)$. Table 4 lists the best fit parameters.

Figure 6 shows the time evolution of the best fit parameters. The most striking change is in the evolution of $m_{0}$, which becomes systematically larger with time (i.e., the companion becomes fainter). The best-fit linear change is $\dot{m}_{0}=0.0131 \pm$

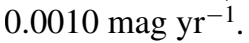

We tested for possible evolution in the orbital period to ensure that phase mis-specification was not the source of the observed decline in $m_{0}$. (The integrated statistical uncertainty from the radio ephemeris on the orbital phase is just $0.16 \mathrm{~s}$ over our ten-year baseline.) The best fit to our data is consistent with no change in the orbital period; the $1 \sigma$ confidence limits are $-2.1 \times 10^{-10}<\dot{P}_{\text {orb }}<2.8 \times 10^{-9}$. Additionally, if we discard all phase information and simply compute the median magnitudes in two-year bins, we see a comparable decline. We conclude that the observed dimming is robust to variations of the orbital period at this scale and assume $\dot{P}_{\text {orb }}=0$ for the remaining discussion.

Despite the clear linear decline in $m_{0}$, secular changes in the other coefficients are less apparent. Our ability to discern shape changes is hindered by the heterogeneous data set, with instruments, phase coverage, and precision varying from year to year. Few of the linear fits to the parameter evolution shown in Figure 6 are formally acceptable; $\chi^{2}$ values for $m_{0}-a_{5}$ are $45.9,17.5,8.0,16.6,25.8$, and 3.0, all for 4 degrees of freedom. All of the coefficients except $a_{4}$ appear generally consistent with a constant value, but with one or two stochastically outlying points that we attribute to the mixed data set.

The behavior the coefficient $a_{4}$ is particularly difficult to interpret. This sine term corresponds to relativistic beaming. For our fit radial velocity amplitude $K=250 \mathrm{~km} \mathrm{~s}^{-1}$, the expected amplitude is $\approx 4$ mmag (Loeb \& Gaudi 2003). However, our fit values are nearly an order of magnitude larger and change sign, implying an physically impossible reversal of the orbital velocity. Moreover, the sine coefficient $a_{5}$ is inconsistent with the value of zero we would expect from a self-consistently modeled light curve (Mazeh \& Faigler 2010).

We also fit Equation (2) to our best-fit binary model from Section 4 . In this case $a_{1}-a_{3}$ took values very similar to those obtained from direct fits to the data, but $a_{4}$ and $a_{5}$ were approximately zero. This suggests that our fit values of $a_{4}$ and $a_{5}$ are modeling (possibly spurious) features of the data not present in the geometric approximation of the photosphere assumed by Icarus. These apparent shape variations are most likely due to the complexities of combining photometry with irregular phase coverage from several distinct surveys.

More speculatively, however, it is possible that we are seeing an additional emission component from outside the companion's Roche lobe-for example, from the intrabinary shock region, if the radio-eclipsing material there is not optically thin, or from a dim remnant accretion disk. In the latter case disk instabilities could be responsible for the observed dimming. Roberts et al. (2015) report that the X-ray emission from PSR J2129-0429 shows an unusual phase dependence, with two strong but asymmetric peaks bracketing the companion superior conjunction, near the quadrature phases. Such a signal could put power into the $a_{4}$ and $a_{5}$ sine coefficients. On the other hand, our optical spectroscopy of the system does not display any features obviously distinct from a stellar companion.

We explored potential physical origins of the observed longterm variation of $m_{0}$. For the companion to get fainter, we could have a change in the radius (becomes smaller), in the effective temperature (becomes cooler), or both. These could be due to perturbation by a past episode of active accretion that occurred before 2003, when our photometry begins. We tested these possibilities by modifying our best-fit binary models.

First, we changed the Roche filling factor (equivalent to changing the radius). As expected, as the filling factor decreases the companion gets fainter, with a fractional decrease of $0.01 \% \mathrm{yr}^{-1}$ required to match the change in $m_{0}$. However, the other coefficients also changed (indeed, we would expect the ellipsoidal modulations to decrease as the filling factor decreases). The most significant change is in $a_{2}$, which would 

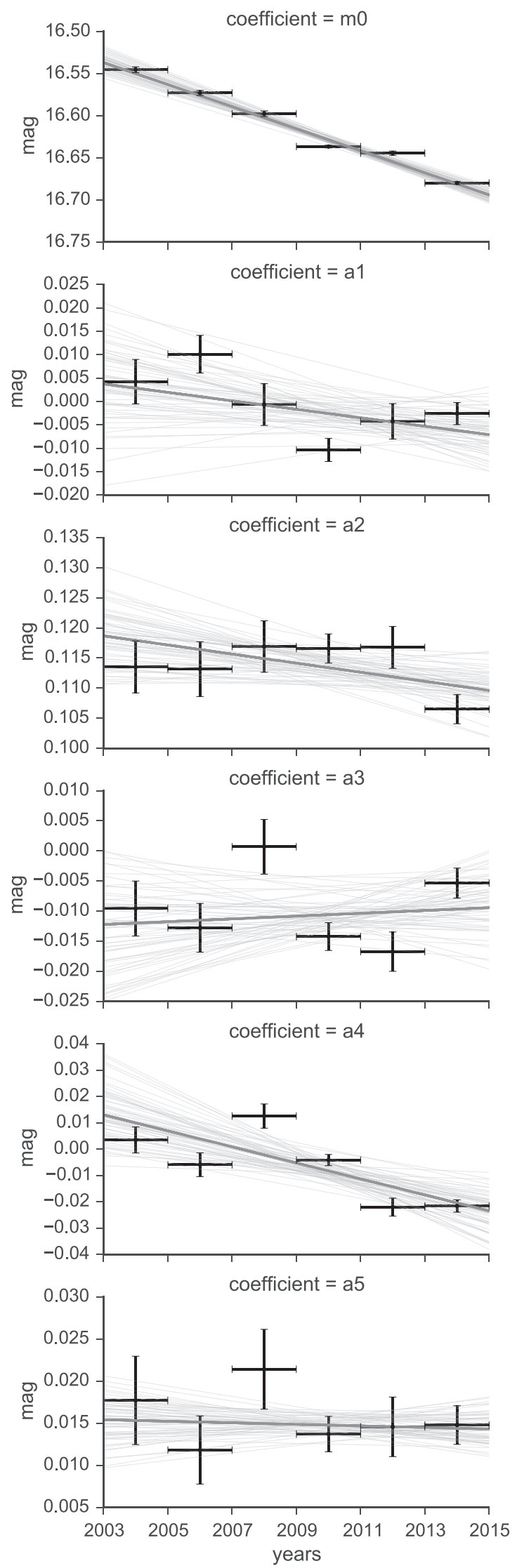

Figure 6. Temporal evolution of the best-fit light curve parameters (Equation (2)). The dark gray lines are the best fit linear model; random deviates drawn from the fit covariance matrix are plotted in light gray.

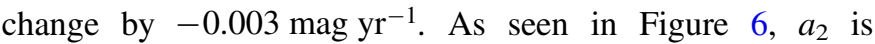
essentially constant except for the final bin. The best-fit line gives $\dot{a}_{2}=-0.0007 \pm 0.0005 \mathrm{mag} \mathrm{yr}^{-1}$, smaller than predicted under the assumption that the flux decline is due to the companion shrinking.

The other simple option would be a change in effective temperature. We decreased both the night-side (base) temperature and day-side temperatures, with the irradiation temperature fixed. This may be overly simplified, if the pulsar's irradiation is changing, but our fits suggest the irradiation term $a_{1}$ is essentially constant. In this model the change in $m_{0}$ requires a decrease of about $10 \mathrm{~K} \mathrm{yr}^{-1}$ in $T_{\text {night, }}$ which also leads to a

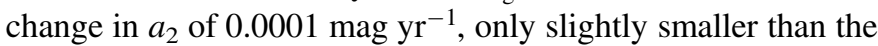
observed value. There is also a decrease in the mean $g-r$

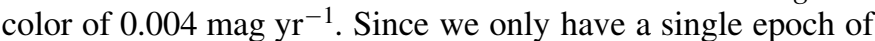
well-sampled multi-band photometry we cannot look for color variations at this time.

While a decreasing effective temperature is most consistent with our current data, the thermal timescale of the star is 10 Myr, much longer than the yearly dimming we observe. Even when limited to the convective envelope, the thermal timescale is 3 Myr.

Despite the clear trend toward dimmer companion magnitudes seen in CRTS, LCOGT, and PTF, we are thus unable to form a self-consistent picture for the physical cause of the variation. Additional multicolor and high-signal-to-noise ratio monitoring in the years ahead should help resolve this ambiguity.

\section{DISCUSSION}

While PSR J2129-0429 clearly meets the observational criteria for identification as a redback system-it is an eclipsing MSP with a nondegenerate companion of a few tenths of a solar mass-its extreme parameters relative to other systems in that population as well as its unusual position in evolutionary phase space make it unique among currently known redbacks.

Our best-fit mass for the non-degenerate companion of PSR J2129-0429 is $M_{\mathrm{c}}=0.44 \pm 0.04 M_{\odot}$, one of the heaviest of known redback systems. (PSR J1723-2837 may have a heavier companion, but its mass is poorly constrained (0.4-0.7 $M_{\odot}$; Crawford et al. 2013).) Combined with its long pulse period (7.6 ms; Hessels et al. 2011) and strong magnetic field $\left(B \sim 1.6 \times 10^{9} \mathrm{G}\right.$; Roberts et al. 2015) relative to other redback systems (Roberts 2013), these data suggest that the PSR J2129-0429 system is early in its recycling phase.

The pulsar companion is $95 \%$ Roche-lobe filling, consistent with a "quasi-Roche lobe overflow" (qRLOF) state where irradiation feedback during a previous accretion phase causes the companion to transfer more mass than it would without irradiation, leaving it just below Roche filling after the cessation of accretion (Benvenuto et al. 2015). As discussed in that paper, however, the qRLOF model cannot account for the ryear timescale variations seen here as well as in the redback systems that have been observed to transition between accreting and detached states.

The orbital period and companion mass of the PSR J2129 -0429 system appear compatible with standard binary evolution of a neutron star and a normal main-sequence companion under some initial conditions (Figure 2 of Podsiadlowski et al. 2002). Interestingly, the bifurcation of evolutionary tracks into short and long period systems occurs near the position of PSR J2129-0429 in the $P_{\text {orb }}-M_{\mathrm{c}}$ plane. 


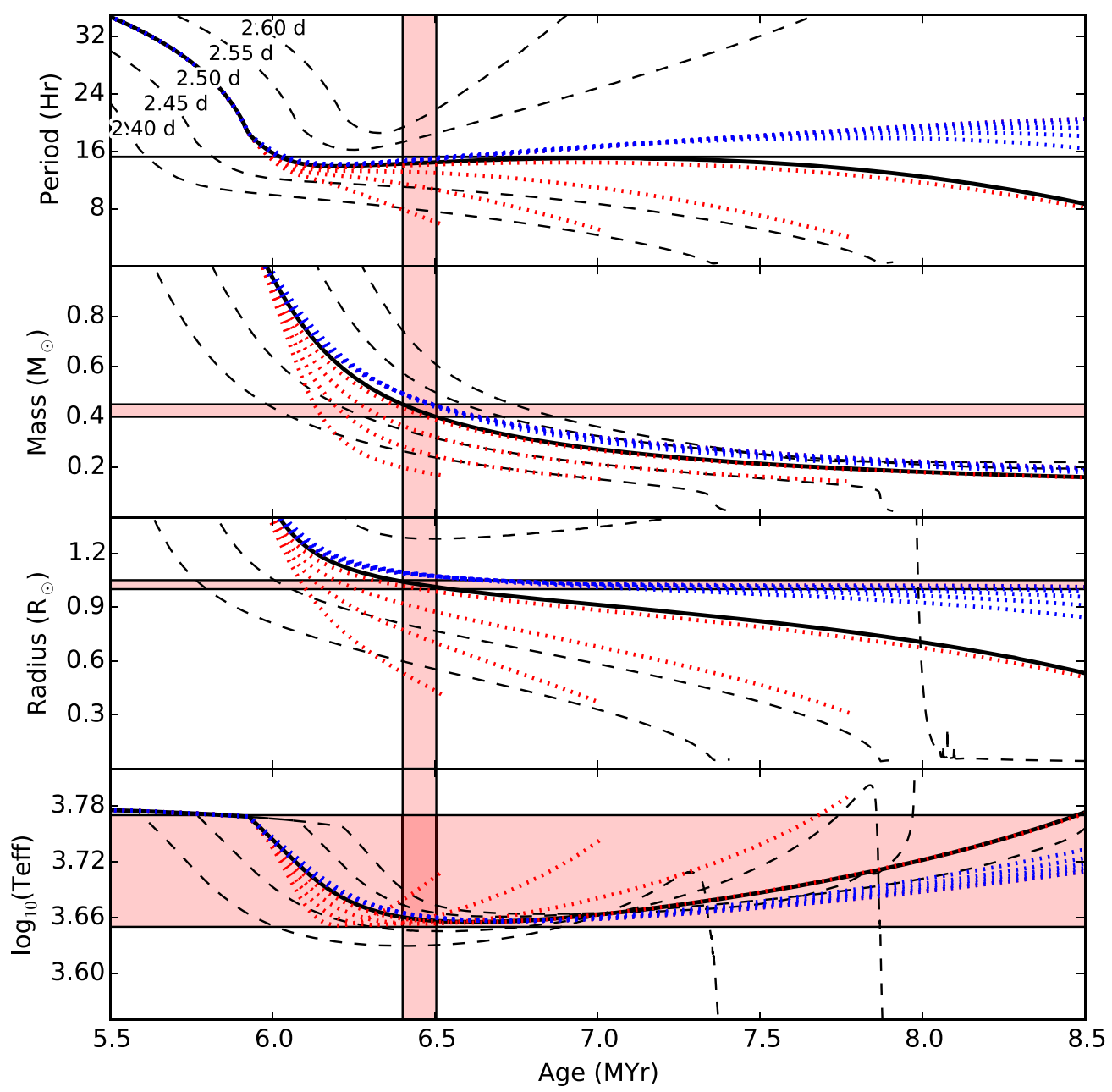

Figure 7. The evolutionary path of the orbital period, donor mass, donor radius, and donor temperature for a $1.143 M_{\odot}$ zero-age main sequence donor orbiting a $1.6 M_{\odot}$ neutron star. The solid lines display the effect of varying the initial orbital period in a range from 2.40 to 2.60 days. The dotted lines show the effects of varying mass loss assuming the 2.50 day initial period. The blue dotted lines display the effect of mass loss at the companion, with $\alpha=0$ and $\beta=0,0.25,0.5,0.75,1$, from top to bottom. The red dotted lines display the effect of mass loss at the neutron star, with $\beta=0$ and $\alpha=0,0.25,0.5,0.75,1$, from top to bottom. Note that the uppermost blue and red lines (e.g., $\alpha, \beta=0$ ) overlap. On each plot, the horizontal red shaded region represents the $68 \%$ probability of the given parameter as inferred from our optical light curve modeling. The vertical red shaded region corresponds to the age compatible with the companion mass for the 2.50 days model.

To explore the possible evolutionary history of PSR J2129 -0429 , we conducted a series of simulations using the Modules for Experiments in Stellar Astrophysics (MESA) code (Paxton et al. 2011, 2013, 2015). Figure 7 shows the evolutionary path of a $1.143 M_{\odot}$ zero-age main sequence donor orbiting a $1.6 M_{\odot}$ neutron star. We examined the effect of varying the initial orbital period in a range from 2.40 to 2.60 days.

Using the notation $\alpha, \beta$ and $\delta$ to refer to the mass fraction lost from the system from the vicinity of the donor as a fast wind, from the vicinity of the accretor, and from a circumbinary coplanar toroid, respectively, we can define the accretion efficiency of the neutron star as $\epsilon=1-(\alpha+\beta+\delta)$ (Tauris $\&$ van den Heuvel 2006). For our first set of simulations, we assumed that $\delta$ is negligible and set $\alpha=0.2$ and $\beta=0.5$, thus implying $\epsilon=0.3$. The rest of the binary evolution parameters were left to the default values implemented in MESA, which uses the prescription from Rappaport et al. (1983) for magnetic braking. Effects of evaporation through irradiation were neglected for reasons that will become apparent later.
Systems with initial orbital periods $\lesssim 2.50$ days are converging (case A evolution; Tauris \& van den Heuvel 2006) and will evolve to become black widows and redbacks. At larger initial orbital separations, mass transfer only sets in on the red giant branch (case B evolution; Tauris \& van den Heuvel 2006) and so these systems will diverge to become pulsar binaries with He-core white dwarf companions. At 2.50 days the PSR J2129-0429 system is marginally diverging, but angular momentum loss due to gravitational wave radiation eventually makes the orbit shrink once the main phase of mass transfer has stopped. We also investigated the effect of varying mass-loss parameters $\alpha$ and $\beta$ for the 2.50 day initial period, which are also displayed in Figure 7.

Given our observational constraints on companion mass, radius, and temperature as well as the orbital period, we can determine which evolutionary tracks are feasible by requiring that they produce consistent observables at a single age. As we can see, our evolution tracks indicate that the initial orbital period must be very close to the bifurcation period and require $\alpha \sim 0.25$, while the effect of $\beta$ is negligible in the considered 

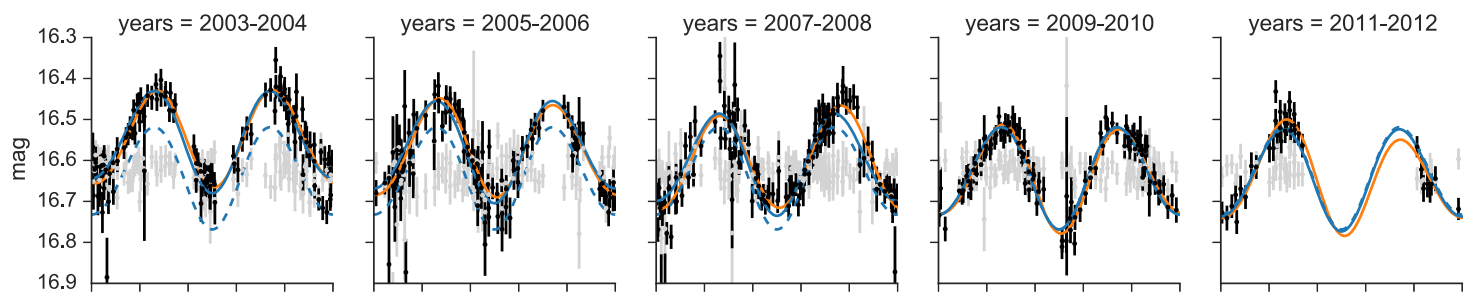

years $=2013-2014$
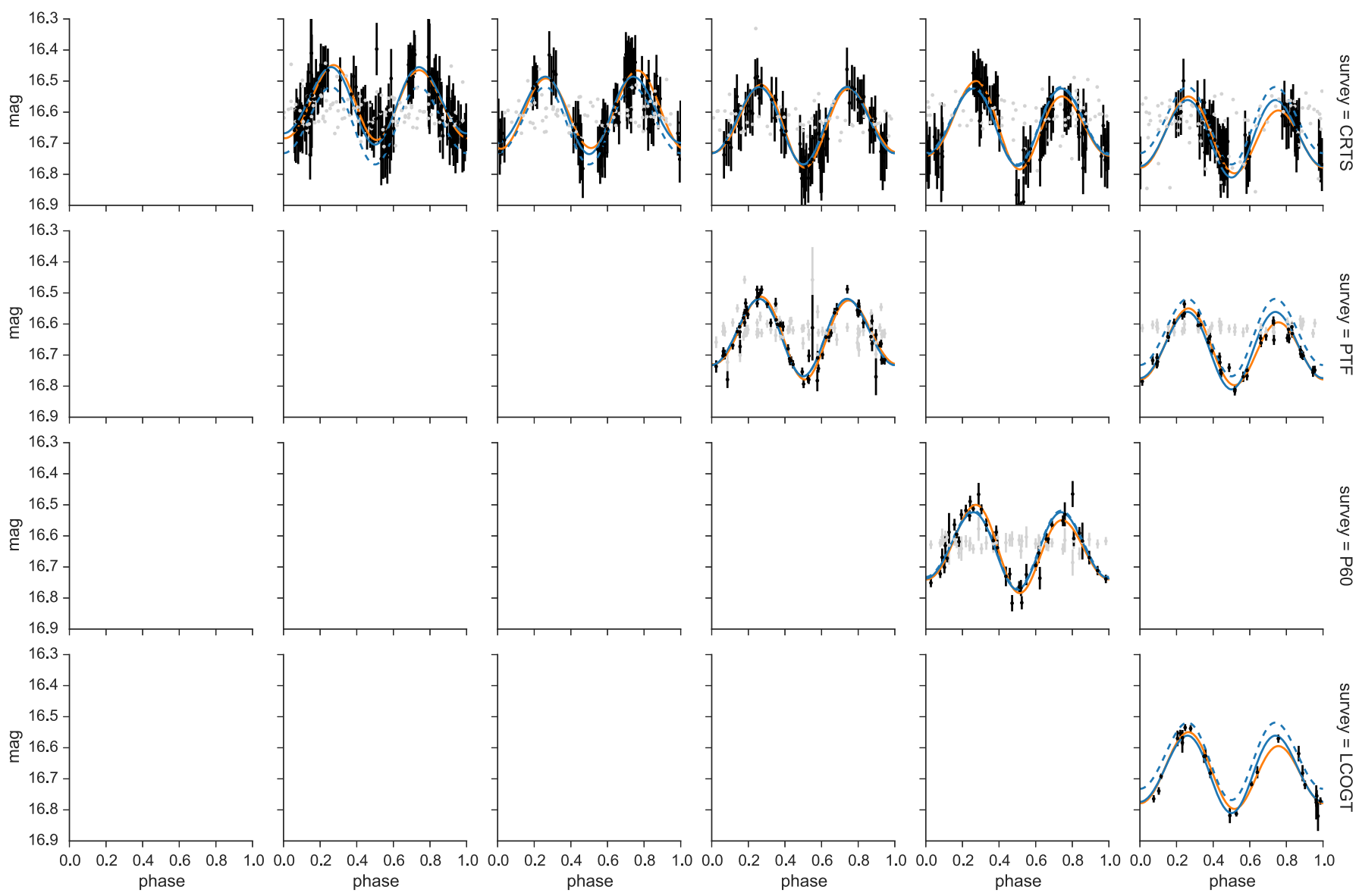

Figure 8. Long-term photometry for PSR J2129-0429 from LINEAR, CRTS, PTF, P60 $\left(r^{\prime}\right)$, and LCOGT $\left(r^{\prime}\right)$. Data are grouped in two-year intervals. Orbital phases are computed from the radio ephemeris. Photometry of PSR J2129-0429 is shown in black, while that of the nearby comparison star is shown in gray. Errors on the CRTS companion photometry are omitted for clarity but are comparable to that of the pulsar. We fit and removed constant offsets from LINEAR ( -0.82 mag), CRTS $(-0.08 \mathrm{mag})$, P60 ( $-0.11 \mathrm{mag})$, and LCOGT $(-0.11 \mathrm{mag})$ to match PTF. Model fits by year with fixed sine and cosine amplitudes are shown in solid blue lines, while the fit to the 2009-2010 data is replicated in blue dashed lines to illustrate the temporal evolution of the source. Model fits by year with the sine and cosine amplitudes free to vary are plotted in orange.

range of evolution. Changing the assumed initial mass ratio qualitatively only changes the timescale of the evolution.

For a reasonable range of initial neutron star masses, the evolution at a similar mass ratio remains unchanged, except for a rescaling of the initial orbital period. In the $M_{\mathrm{NS}, \mathrm{i}}=1.6 M_{\odot}$ scenario, the accreted mass is $\sim 0.2 M_{\odot}$, which implies a neutron star mass of $1.8 M_{\odot}$, similar to the value inferred from our light curve modeling. While we have not searched for compatible solutions for an exhaustive range for parameters at other mass ratios, we found that the mass ratio needs to be close to the presented one if $\alpha=0.2$ and $\beta=0.5$. In every case, we found that the only systems able to reproduce the observed properties are always close to the bifurcation period.

In light of our binary evolution work, we conclude that PSR J2129-0429 is in a very specific region of the phase space. At present the system is close to Roche-lobe filling and thus given its mass could not have been in a significantly tighter orbit in the past. Irradiation from the pulsar is very mild because of the large orbital separation and, despite the fact that it could have been larger in the past when the pulsar was spinning faster, it is unlikely that it has ever been very effective. Indeed, as demonstrated by Chen et al. (2013), evaporation through irradiation leads to an increase of the orbital period and so this mechanism could not have happened significantly given that the current orbit is close to the shortest separation that it has ever been. This also justifies our assumption to neglect the effect of irradiation.

The fate of PSR J2129-0429 is ambiguous: it lies right at the boundary between systems that will become black widows and those that will widen and form a He-core white dwarf binary. Gravitational wave radiation should cause the system to shrink, but if additional mass transfer takes place it will widen the orbit. As it is, the orbit might still be marginally widening if residual mass transfer is still occurring. Whether the orbit 
becomes significantly compact within the next billion years or so depends on the relative rates of expansion and coalescence and whether further episodic mass transfer and irradiation will occur. The balancing of these effects is influenced by the assumed initial mass ratio, which sets the timescale within which this competition may take place.

Our modeling shows that it is possible to produce the observed high pulsar mass $\left(M_{\mathrm{NS}}=1.74 \pm 0.2 M_{\odot}\right)$ with some assumptions under standard recycling scenarios. Other evolutionary scenarios are not ruled out, however. Production of a NS with this mass via Accretion Induced Collapse rather than recycling appears possible, but would require a rapidly rotating WD and high accretion efficiency (Smedley et al. 2015). Another possibility is that the neutron star was born heavy, with a mass near the observed $1.74 M_{\odot}$ (Tauris et al. 2011).

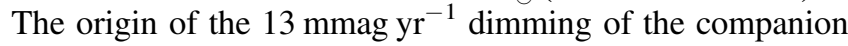
of PSR J2129-0429 discovered in this work remains uncertain. If the companion is shrinking, we would expect to see larger changes in the ellipsoidal modulation of the phased light curve than are observed. Cooling of the companion is compatible with these data, but the expected thermal timescales for the system are much longer than the observed yearly variations. Future multi-color observations will be valuable in monitoring the ongoing evolution of this unique system. Further investigation may reveal if PSR J2129-0429 will eventually destroy its companion or if-like the katipo spider found in New Zealand-it is a redback that shuns cannibalism.

T.A.P., E.C.B., and S.T. acknowledge partial support through NASA Grant No. NNX12AO76G. R.P.B. has received funding from the European Union Seventh Framework Programme under grant agreement PIIF-GA-2012-332393. J.W.T.H. acknowledges funding from an NWO Vidi fellowship and ERC Starting Grant "DRAGNET" (337062). E.C.B. and T.A.P. thank the Aspen Center for Physics and the NSF Grant \#1066293 for hospitality during the editing of this paper.

This paper is based in part on observations obtained with the Palomar 48 inch Oschin telescope and the robotic Palomar 60inch telescope at the Palomar Observatory as part of the Palomar Transient Factory project, a scientific collaboration among the California Institute of Technology, Columbia University, Las Cumbres Observatory, the Lawrence Berkeley National Laboratory, the National Energy Research Scientific Computing Center, the University of Oxford, and the Weizmann Institute of Science; and the Intermediate Palomar Transient Factory project, a scientific collaboration among the California Institute of Technology, Los Alamos National Laboratory, the University of Wisconsin, Milwaukee, the Oskar Klein Center, the Weizmann Institute of Science, the TANGO Program of the University System of Taiwan, and the Kavli Institute for the Physics and Mathematics of the universe.

The CSS survey is funded by the National Aeronautics and Space Administration under Grant No. NNG05GF22G issued through the Science Mission Directorate Near-Earth Objects Observations Program. The CRTS survey is supported by the U.S. National Science Foundation under grants AST-0909182, AST-1313422, and AST-1413600. This work makes use of observations from the Las Cumbres Observatory Global Telescope Network.

Facilities: Hale (Double Beam Spectrograph), PO:1.2 m (Palomar Transient Factory), PO:1.5 m, LCOGT.

\section{REFERENCES}

Abdo, A. A., Ackermann, M., Ajello, M., et al. 2010, ApJS, 188, 405 Ackermann, M., Ajello, M., Allafort, A., et al. 2012, ApJ, 753, 83 Alam, S., Albareti, F. D., Allende Prieto, C., et al. 2015, ApJS, 219, 12

Alpar, M. A., Cheng, A. F., Ruderman, M. A., \& Shaham, J. 1982, Natur, 300,728

Antoniadis, J., Freire, P. C. C., Wex, N., et al. 2013, Sci, 340, 448

Archibald, A. M., Stairs, I. H., Ransom, S. M., et al. 2009, Sci, 324, 1411

Bailes, M., Bates, S. D., Bhalerao, V., et al. 2011, Sci, 333, 1717

Bassa, C. G., Patruno, A., Hessels, J. W. T., et al. 2014, MNRAS, 441, 1825

Bassa, C. G., van Kerkwijk, M. H., Koester, D., \& Verbunt, F. 2006, A\&A, 456, 295

Benvenuto, O. G., De Vito, M. A., \& Horvath, J. E. 2014, ApJL, 786, L7 Benvenuto, O. G., De Vito, M. A., \& Horvath, J. E. 2015, ApJ, 798, 44 Bertin, E., \& Arnouts, S. 1996, A\&AS, 117, 393

Bhalerao, V. B., van Kerkwijk, M. H., \& Harrison, F. A. 2012, ApJ, 757, 10 Bhattacharya, D., \& van den Heuvel, E. P. J. 1991, PhR, 203, 1

Breton, R. P., van Kerkwijk, M. H., Roberts, M. S. E., et al. 2013, ApJ, 769, 108

Cenko, S. B., Fox, D. B., moon, D.-S., et al. 2006, PASP, 118, 1396

Chen, H.-L., Chen, X., Tauris, T. M., \& Han, Z. 2013, ApJ, 775, 27

Cordes, J. M., \& Lazio, T. J. W. 2002, arXiv:astro-ph0207156

Crawford, F., Lyne, A. G., Stairs, I. H., et al. 2013, ApJ, 776, 20

Deller, A. T., Archibald, A. M., Brisken, W. F., et al. 2012, ApJL, 756, L25

Deloye, C. J., \& Bildsten, L. 2003, ApJ, 598, 1217

Demorest, P. B., Pennucci, T., Ransom, S. M., Roberts, M. S. E., \& Hessels, J. W. T. 2010, Natur, 467, 1081

Drake, A. J., Djorgovski, S. G., Mahabal, A., et al. 2009, ApJ, 696, 870

Drake, A. J., Graham, M. J., Djorgovski, S. G., et al. 2014, ApJS, 213, 9

Freire, P. C. C., Bassa, C. G., Wex, N., et al. 2011, MNRAS, 412, 2763

Fruchter, A. S., \& Goss, W. M. 1992, ApJL, 384, L47

Fruchter, A. S., Stinebring, D. R., \& Taylor, J. H. 1988, Natur, 333, 237

Hessels, J. W. T., Roberts, M. S. E., McLaughlin, M. A., et al. 2011, in AIP Conf. Ser. 1357, Radio Pulsars: An Astrophysical Key to Unlock the Secrets of the Universe, ed. M. Burgay et al. (Melville, NY: AIP), 40

IAU SOFA Board 2015, IAU SOFA Software Collection, http://www. iausofa.org

Kaplan, D. L., Bhalerao, V. B., van Kerkwijk, M. H., et al. 2013, ApJ, 765, 158 King, A. R., Davies, M. B., \& Beer, M. E. 2003, MNRAS, 345, 678

Kluzniak, W., Ruderman, M., Shaham, J., \& Tavani, M. 1988, Natur, 334, 225

Laher, R. R., Surace, J., Grillmair, C. J., et al. 2014, PASP, 126, 674

Lattimer, J. M. 2012, ARNPS, 62, 485

Law, N. M., Kulkarni, S. R., Dekany, R. G., et al. 2009, PASP, 121, 1395

Levinson, A., \& Eichler, D. 1991, ApJ, 379, 359

Li, M., Halpern, J. P., \& Thorstensen, J. R. 2014, ApJ, 795, 115

Loeb, A., \& Gaudi, B. S. 2003, ApJL, 588, L117

Lucy, L. B. 1967, ZAp, 65, 89

Mazeh, T., \& Faigler, S. 2010, A\&A, 521, L59

Munari, U., Sordo, R., Castelli, F., \& Zwitter, T. 2005, A\&A, 442, 1127

Nice, D. J., Splaver, E. M., Stairs, I. H., et al. 2005, ApJ, 634, 1242

Nolan, P. L., Abdo, A. A., Ackermann, M., et al. 2012, ApJS, 199, 31

Ofek, E. O., Frail, D. A., Breslauer, B., et al. 2011, ApJ, 740, 65

Ofek, E. O., Laher, R., Law, N., et al. 2012, PASP, 124, 62

Oke, J. B., \& Gunn, J. E. 1982, PASP, 94, 586

Papitto, A., Ferrigno, C., Bozzo, E., et al. 2013, Natur, 501, 517

Patruno, A., Archibald, A. M., Hessels, J. W. T., et al. 2014, ApJL, 781, L3

Paxton, B., Bildsten, L., Dotter, A., et al. 2011, ApJS, 192, 3

Paxton, B., Cantiello, M., Arras, P., et al. 2013, ApJS, 208, 4

Paxton, B., Marchant, P., Schwab, J., et al. 2015, arXiv:1506.03146

Phinney, E. S., Evans, C. R., Blandford, R. D., \& Kulkarni, S. R. 1988, Natur, 333,832

Phinney, E. S., \& Kulkarni, S. R. 1994, ARA\&A, 32, 591

Podsiadlowski, P., Rappaport, S., \& Pfahl, E. D. 2002, ApJ, 565, 1107

Radhakrishnan, V., \& Srinivasan, G. 1982, CSci, 51, 1096

Rahmer, G., Smith, R. M., Bui, K., et al. 2012, Proc. SPIE, 8446, 2

Rappaport, S., Verbunt, F., \& Joss, P. C. 1983, ApJ, 275, 713

Rau, A., Kulkarni, S. R., Law, N. M., et al. 2009, PASP, 121, 1334

Roberts, M. S. E. 2011, in AIP Conf. Ser. 1357, Radio Pulsars: An Astrophysical Key to Unlock the Secrets of the Universe, ed. M. Burgay et al. (Melville, NY: AIP), 127

Roberts, M. S. E. 2013, in IAU Symp. 291, Neutron Stars and Pulsars: Challenges and Opportunities after 80 Years, ed. J. van Leeuwen (Cambridge: Cambridge Univ. Press), 127

Roberts, M. S. E., McLaughlin, M. A., Gentile, P., et al. 2015, arXiv: 1502.07208 
Romani, R. W., Filippenko, A. V., Silverman, J. M., et al. 2012, ApJL, 760, L36

Schlafly, E. F., \& Finkbeiner, D. P. 2011, ApJ, 737, 103

Schroeder, J., \& Halpern, J. 2014, ApJ, 793, 78

Sesar, B., Grillmair, C. J., Cohen, J. G., et al. 2013, ApJ, 776, 26

Sesar, B., Stuart, J. S., Ivezić, Ž, et al. 2011, AJ, 142, 190

Smedley, S. L., Tout, C. A., Ferrario, L., \& Wickramasinghe, D. T. 2015, MNRAS, 446, 2540

Stappers, B. W., Archibald, A. M., Hessels, J. W. T., et al. 2014, ApJ, 790, 39

Stokes, G. H., Evans, J. B., Viggh, H. E. M., Shelly, F. C., \& Pearce, E. C. 2000, Icar, 148, 21
Strader, J., Chomiuk, L., Cheung, C. C., et al. 2015, arXiv:1502.05999

Tauris, T. M., Langer, N., \& Kramer, M. 2011, MNRAS, 416, 2130

Tauris, T. M., \& van den Heuvel, E. P. J. 2006, in Formation and Evolution of Compact Stellar X-ray Sources, ed. W. H. G. Lewin, \& M. van der Klis (Cambridge: Cambridge Univ. Press), 623

The Fermi-LAT Collaboration 2015, arXiv:1501.02003

Tody, D. 1986, Proc. SPIE, 627, 733

van den Heuvel, E. P. J., \& van Paradijs, J. 1988, Natur, 334, 227

van Kerkwijk, M. H., Breton, R. P., \& Kulkarni, S. R. 2011, ApJ, 728, 95

York, D. G., Adelman, J., Anderson, J. E., Jr., et al. 2000, AJ, 120 1579 